\title{
Data envelopment analysis for analyzing technical efficiency in aquaculture: the bootstrap methods
}

\author{
Le Kim Long*: Faculty of Economics, Nha Trang University, Vietnam \\ Postal address: Faculty of Economics, NhaTrang University, 02 Nguyen Dinh Chieu, Nha Trang, Vietnam. \\ Email address: lekimlong@gmail.com; lekimlong@ntu.edu.vn
}

Le Van Thap: Faculty of Economics, Nha Trang University, Vietnam and School of Economics, University of Economics, HCM City, Vietnam

Postal address: Faculty of Economics, Nha Trang University, 02 Nguyen Dinh Chieu, Nha Trang, Vietnam. E-mail address: thapdhts@yahoo.com; thaplv@ntu.edu.vn

Nguyen Trong Hoai: School of Economics Development, University of Economics, HCM City, Vietnam Postal address: 59 C Nguyen Dinh Chieu Dist 3, HCM City, Vietnam.

Email address: hoaianh@ueh.edu.vn

Thuy Thi Thanh Pham: Faculty of Economics, Nha Trang University, Vietnam and The Actic University, Tromso, Norway

Postal address: Postboks 6050 Langnes, 9037 Troms $\emptyset$

E-mail address: thanh.thuy@uit.no

* Corresponding author 


\begin{abstract}
The paper adopts the bootstrap procedures proposed by Simar and Wilson for data envelopment analysis to analyze technical efficiency in aquaculture. The data of 318 intensive shrimp farming households in the south-central coast of Vietnam is used as a case for analysis. The result demonstrates that the null hypothesis of constant returns-to-scale is rejected in favour of variable returns-to-scale for the production technology. Moreover, the potential improvement is certainly greater using bootstrapping than that using the conventional data envelopment analysis, which has been adopted widely in the aquaculture literature for technical efficiency estimation. By adopting the double bootstrap proposed by Simar \& Wilson (2007), the bias-corrected technical efficiency is 0.69 , and at the $95 \%$ confidence interval with the lower limit of 0.65 and the upper limit of 0.75 . In addition, factors that might statistically positive influence technical efficiency in this farming are larger farm size, having access to formal credit; whereas negative influences are cultural length.
\end{abstract}

Key words: bootstrap, DEA, intensive shrimp farming, technical efficiency, Vietnam

\title{
1. INTRODUCTION
}

The world is faced with a critical challenge of feeding the growing population that is expected to reach 9.6 billion by 2050 . An increase in food production must take place in a context where resources necessary for food production, such as land and water, are even scarcer in a more crowded world, whereas the world is required to change the ways it conducts economic activities in the face of global climate change (Kobayashi et al., 2015). Efficient input utilization in food production is, therefore, among major concerns aiming sustainably supply food for growing population in the world.

Aquaculture has seen a dramatic growth globally over the last decades. In 1974, aquaculture provided only $7 \%$ of all fish for human consumption, whereas in 2015 the percentage had increased to $45 \%$. Total aquaculture production reached 106 million tons, worth US\$163 billion, of which crustaceans, mollusks and other non-fish animals made up one third (FAO, 2018). As most of the wild stocks are either fully 
exploited or overexploited, aquaculture is expected to overtake wild capture fisheries in terms of food production and to play a key future role in the supply of food for a rising world population. Kobayashi et al., (2015) predict that the total fish supply will rise up to 186 million tons in 2030, and aquaculture is entirely responsible for the increase. The fastest aquaculture growth is expected for tilapia and shrimp, whereas India, Latin America, Caribbean and Southeast Asia are expected to be the largest aquaculture expanding countries (Kobayashi et al., 2015).

Vietnam is a country in Southeast Asia and one of the leading shrimp exporters in the world. Its white-leg shrimp farming in brackish water has become a major part of coastal economic development since 2001. White-leg shrimp aquaculture, mainly in the central provinces of Vietnam and the Mekong river delta, grew from 13,455 ha in 2005 to 94,246 ha in 2016 (Fisheries Directorate of Vietnam, 2017). By 2014, the whiteleg shrimp production achieved 324,581 tons, reaching the export value of 2.3 billion USD. This accounts for 35\% of Vietnam's total export value of fisheries (Long et al., 2016). The rapid growth of white-leg shrimp farming had put this aquaculture faces several severe risks, especially the spread of shrimp disease and pollution (Anh et al., 2010; Thuyet et al., 2012; and Long et al., 2016). These problems were also found in some other fast growing cultured species (see Kobayashi et al., 2015 for detail). Moreover, some shrimp farmers experience financial losses. Thirty percent of shrimp farms in the Mekong river delta experienced financial losses in 2006 (Sinh, 2006). The same situation was also hold for many shrimp farms in Khanh Hoa (Long and Binh, 2013). Den et al. (2007), researching shrimp farms in Bac Lieu province, found that the less technically efficient the farms are, the more likely they are experience of financial losses.

To meet the increasing protein demand due to population growth and an increase in household earning, it is, therefore, important to improve the technical efficiency (optimal use of inputs) of their production. This, firstly, will lead to lower production costs per unit and higher returns to shrimp farmers. Secondly, saving production input such as feed, chemical and power are very important for mitigating harmful emissions to our air, land and water. This is not only due to environmental concerns, but also long-term development of 
shrimp farming (see Long et al. 2016 for details). Thus, this study aims to estimate the technical efficiency and determine the factors responsible for technical efficiency in this intensive shrimp farming.

Data envelopment analysis (DEA), a nonparametric technique adopting a linear programming approach, is a leading analytical technique for analyzing technical efficiency. This technique is favourable because it does not impose a priori functional form and allows for multiple output technologies (Badunenko \& Mozharovskyi, 2016). There are, however, not many studies using DEA for analyzing technical efficiency in aquaculture pay much attention to its statistical properties (see Sharma \& Lueng, 2003 and Iliyasu et al., 2014).

The paper extends previous studies by adopting the bootstrap procedures proposed by Simar and Wilson (1998, 2000 \& 2007) for DEA to make statistical inferences about technical efficiency. The results from the bootstrap methods are also presented in comparison with the conventional DEA approach. The case of white-leg shrimp farming in the south-central coast of Vietnam is used for an analysis. The main objectives of this study are as follows: (i) applying Simar and Wilson's (2002) returns-to-scale test for the production technology of shrimp farming; (iii) comparing the technical efficiencies estimated by the conventional DEA and the bootstrap methods; (iii) adopting the double bootstrap procedure (Algorithm 2) developed by Simar and Wilson (2007) to estimate and to identify factors that are significant in explaining differences in levels of technical efficiency between shrimp farms.

\section{LITERATURE REVIEW}

Technical efficiency, developed by Farrell (1957) relates to the degree to which a farm produces the maximum feasible output from a given bundle of inputs, or uses the minimum feasible amount of inputs to produce a given level of output. The conventional DEA method has been adopted for technical efficiency analysis in aquaculture studies for many years (see for reviews see Sharma \& Leung, 2003 and Iliyasu et al., 2014). However, it is rather difficult for statistical inferences about technical efficiency when applying DEA method as following reasons. Firstly, all estimates of technical efficiency are sample specific. 
Although the DEA method is to be conventional, the efficiency is still computed relatively to estimated and not true frontier. The efficiency scores obtained from a finite sample are subject to sampling variation of the estimated frontier (Simar \& Wilson, 1998 and Badunenko \& Mozharovskyi, 2016). Secondly, the estimated technical efficiency measures are too optimistic, due to the fact that the DEA estimate of the production set is necessarily a weak subset of the true production set under standard assumptions underlying DEA (Simar \& Wilson, 2000 and Badunenko \& Mozharovskyi, 2016). Therefore, it should be careful when attempting to draw broad policy implications in aquaculture from a specific set of related but independent studies.

To overcome these, Simar and Wilson $(1998,2000)$ have developed the smoothed bootstrap procedure into the DEA framework to introduce the statistical foundation of nonparametric frontier model. To implement the bootstrap into DEA framework, assuming that the original sample data is generated by the data generation process (DGP) and that the DGP by taking a 'new' or pseudo data set that is drawn from the original data set can be stimulated. The DEA model, then, is re-estimated with this 'new' data. By repeating this process many times, an empirical distribution of these bootstrap values can be derived. This gives a Monte Carlo approximation of the sampling distribution and facilitates inference procedures. Their single bootstrap method, based on statistical well-defined models, allows for consistent estimation of the production frontier, corresponding technical efficiencies, as well as standard errors, confidence intervals and hypothesis testing. Despite this development, the application of this technique for estimating Farrell (1957)'s technical efficiency has thus far been limited in aquaculture. For our best knowledge, there are only the works of Chang et al. (2010), Asche et al. (2013), Iliyasu et al. (2016), Lam Anh et al. (2018) and Hai Au et al. (2018) used the single bootstrap for correcting bias in estimation of technical efficiency (see Sharma \& Lueng, 2003 and Iliyasu et al., 2014 for reviewing technical efficiency studies adopting DEA in aquaculture before the year of 2010; and Table 1 below for this decade).

[Insert Table 1 about here] 
The returns-to-scale for the production technology is an important issue in technical efficiency analysis. According to Coelli et al. (2005), the constant returns-to-scale (CRS) DEA model is only appropriate when the farm is operating at an optimal scale. Factors such as imperfect competition, constraints on finance, socio-economic limitations of the aquaculture farmers may cause the farm to not operate at an optimal scale in practice (see for examples, Alam \& Murshed-e-Jahan, 2008; Nielsen, 2011 for a detailed discussion). Therefore, the variable returns-to-scale (VRS) DEA model for production technology is often assumed in aquaculture studies, especially for the cases of aquaculture farming in developing countries (for examples, see Kiet \& Fisher, 2014; Zongli et al., 2017; Lam Anh et al., 2018; and Hai Au et al., 2018). However, these studies only adopt the assumption of variable returns-to-scale production technology without testing. Simar and Wilson (2002) showed that a false assumption about constant returns-to-scale may lead to inconsistent efficiency scores, and a false assumption about variable returns-to-scale may cause a loss in statistical efficiency. Based on the single bootstrap procedure, Simar and Wilson (2002) proposed returns-to-scale tests to raise the precision in the measurement of the boundary of the production possibilities set in the nonparametric analysis (see Bogetoft \& Otto, 2010 for details and Besstremyannaya, 2013 for an empirical application). We, therefore, apply Simar \& Wilson's (2002) returns-to-scale test to raise the precision in the measurement of the boundary of the production possibilities set in the non-parametric analysis.

Apart from the technology used in aquaculture, individual farm characteristics may also have an impact on technical efficiency. For example, farmers not participating training in shrimp farming techniques are expected to perform worse than those who do (provided that they are operating under otherwise similar conditions). Iliyasu et al. (2014) recognized that the majority of aquaculture studies use a conventional twostage DEA approach, in which efficiency is estimated in the first stage, and then the estimated efficiencies are regressed on the individual farm characteristics with Tobit regression in the second stage (see Iliyasu et al., 2014 and Table 1). Following the McDonald (2009)'s argument that technical efficiency scores were fraction data, not censored data, Iliyasu \& Mohamed (2015) and Iliyasu et al. (2016) have, therefore, adopted ordinary least square (OLS) method in the second stage of DEA approach for the cases of 
aquaculture in Malaysia. As existing aquaculture studies undertaking efficiency analysis using conventional two-stage DEA have not considered its statistical properties, we need to be careful when attempting to draw broad policy implications based on their findings. Simar and Wilson (2007) criticized the conventional twostage DEA approach because its estimates are biased and serially correlated, therefore invalidating conventional inferences in the second stage.

To resolve this problem, Simar and Wilson (2007) developed two complementary consistent procedures in the two-stage DEA approach: Algorithm 1 (the single bootstrap truncated regression) and Algorithm 2 (the double bootstrap DEA). Algorithm 2 is preferred over Algorithm 1 (see Simar and Wilson, 2007 and Fernandes at el., 2018 for detailed discussions). The double bootstrap procedure enables consistent inference within models explaining efficiency scores, while simultaneously producing standard errors and confidence intervals for these efficiency scores (Simar \& Wilson, 2007, $2011 \&$ 2013). This double bootstrap technique has been applied empirically to several studies in agriculture and other sectors such as crop and livestock farms in Czech Republic (Latruffe et al., 2008); rice farming in Bangladesh (Balcombe et al., 2008); agriculture farms in the US (Olson and Vu, 2009); the Greek poultry sector (Keramidou and Mimis, 2011); the peripheral European domestic banks (Fernandes et al., 2018). However, few studies adopt this approach in aquaculture. Lam Anh et al. (2018), Hai Au et al. (2018) and Anh Ngoc et al. (2018) have adapted the Algorithm 1, thus enabling statistical inference in the second-stage regression.

For our best knowledge, there is only Thap et al. (2016) adopting Algorithm 2 in aquaculture studies. The reason may be due to computational burden of the complex procedure and sample size (see also Simar and Wilson, 2007; Singbo et al., 2010 and 2014). To fulfill this gap, this paper will employ this Algorithm 2 with a double bootstrap procedure that gives confidence intervals for the efficiency estimate and also enables consistent inference within models explaining technical efficiency.

\section{METHODOLOGY}

\subsection{The Models}




\subsubsection{The conventional DEA}

Technical efficiency relates to the degree to which a farm produces the maximum feasible output from a given bundle of inputs, or uses the minimum feasible amount of inputs to produce a given level of output. These two definitions of technical efficiency lead to what are known as output-oriented and input-oriented efficiency measures respectively. Orientation (input or output) is selected according to which factors farmers have most control over (see also Nielsen, 2011). In this study, an input-oriented DEA model was used. The input orientation approach to DEA for efficiency analysis has also been employed by some aquaculture studies for developing countries, such as those of Alam \& Murshed-e-Jahan (2008), Alam (2011), Kiet \& Fisher (2014), Zongli et al. (2017), Lam Anh et al. (2018) and Hai Au et al. (2018). The orientation aims to reduce the input amount by as much as possible while keeping at least the present output level. This is a sensible assumption for white-leg shrimp farmers in developing countries, like Vietnam, where reducing production cost is very important due to their limited finances. For the $j^{\text {th }}$ farm out of $\mathrm{N}$ farms, the input-based technical efficiency index for VRS DEA framework, $T E_{j}$, is defined as:

$$
T E_{j}=\widehat{\theta}_{J}=\min \left\{\theta_{j} \mid y_{j} \leq Y \lambda ; \theta_{j} x_{j} \geq X \lambda ; I \lambda=1\right\}
$$

where $y_{j}$ and $x_{j}$ are respectively the output and input matrices of the $j^{\text {th }}$ farm; $\mathrm{Y}$ and $\mathrm{X}$ are respectively the

output and input matrices of the sample; $T E_{j}=\widehat{\theta}_{J}$ is the technical efficiency value ranging from 0 to $1, \lambda$ is a vector of constant weights that defines the linear combination of the peers of the $j^{\text {th }}$ farm, and $\sum_{j=1}^{n} \lambda_{j}=1$ for assuming VRS (see e.g. Coelli, 2005 for details). For explaining the sources of farm level efficiency, the TE score calculated from (1) for each farm, censored between zero and one, are often regressed on the individual farm characteristics with Tobit regression (see e.g. Kiet \& Fisher, 2014 and Zongli et al.. 2016).

\subsubsection{The single bootstrap proposed by Simar \& Wilson (1998, 2000)}

The single bootstrap, based on the smoothed bootstrap procedure, proposed by Simar \& Wilson (1998, 2000) for input-oriented VRS DEA is presented in details as follows.

Step1: Compute the estimate of technical efficiency, $\hat{\theta}_{j}$, for the $j^{t h}$ farm as in equation (1) 
Step 2: Use bootstrap via smooth sampling from $\widehat{\theta_{1}}, \ldots, \widehat{\theta_{N}}$ to obtain a bootstrap replica $\theta_{1}^{*}, \ldots, \theta_{N}^{*}$. This is done as follows

(2.1) Bootstrap, sample with replacement from $\widehat{\theta_{1}}, \ldots, \widehat{\theta_{N}}$, and call the results $\beta_{1}, \ldots, \beta_{N}$

(2.2) Simulate standard normal independent random variables $\epsilon_{1}, \ldots, \epsilon_{N}$

(2.3) Calculate $\widetilde{\theta}_{J}=\left\{\begin{array}{c}\beta_{j}+h \epsilon_{j} \text { if } \beta_{j}+h \epsilon_{j} \leq 1 \\ 2-\beta_{j}-h \epsilon_{j} \text { otherwise }\end{array}\right.$. Note that $\widetilde{\theta}_{J} \leq 1$ and $h$ is the window or band width.

(2.4) Adjust $\widetilde{\theta_{J}}$ to obtain parameters with asymptotically correct variance, and then estimate the variance $\widehat{\sigma^{2}}=\frac{1}{N} \sum_{j=1}^{N}\left(\widehat{\theta_{J}}-\overline{\hat{\theta}}\right)^{2}$ and calculate $\theta_{j}^{*}=\bar{\beta}+\frac{1}{\sqrt{1+h^{2} / \widehat{\sigma^{2}}}}\left(\widetilde{\theta_{J}}-\bar{\beta}\right)$ where $\bar{\beta}=\frac{1}{N} \sum_{j=1}^{N} \beta_{j}$

Step 3: For $j=1, \ldots, \mathrm{N}$, a pseudo data set of $\left(x_{j, b}^{*}, y_{j, b}^{*}\right)$ where $x_{j, b}^{*}=\left(\widehat{\theta}_{j} / \theta_{j}^{*}\right) x_{j}$ and $y_{j, b}^{*}=y_{j}$. Calculate the new DEA score $\widehat{\theta_{J}^{*}}$ for each farm from equation (1) by taking the pseudo data as a reference.

Step 4: Repeat steps (1) to (3) for B times to yield B new DEA TE scores $\widehat{\theta_{J}^{*}}$ for $j=1, \ldots, \mathrm{N}$. Therefore, the bias-corrected estimator of $\hat{\theta}_{j}$ can be computed as $\widehat{\hat{\theta}}_{J}=B^{-1} \sum_{b=1}^{B} \widehat{\theta_{J}^{*}}$

Step 5: The confidence interval of a $(1-\alpha)$ level for the technical efficiency can be established by finding value $a_{\alpha}, b_{\alpha}$ such that $\operatorname{Pr}\left(-a_{\alpha} \leq \hat{\theta}_{j}-\theta_{j} \leq-b_{\alpha}\right)=1-\alpha$. Since we do not know the distribution of $\left(\hat{\theta}_{j}-\right.$ $\left.\theta_{j}\right)$, we can use the bootstrap values to find $\widehat{a_{\alpha}}, \widehat{b_{\alpha}}$ such that $\operatorname{Pr}\left(-\widehat{a_{\alpha}} \leq \widehat{\theta_{J}^{*}}-\widehat{\theta}_{j} \leq-\widehat{b_{\alpha}}\right)=1-\alpha$. Therefore the estimated confidence level of $(1-\alpha)$ for technical efficiency of $j^{\text {th }}$ farm is $\widehat{\theta_{J}}+\widehat{b_{\alpha}} \leq \theta_{j} \leq$ $\widehat{\theta}_{j}+\widehat{a_{\alpha}}$

Next, adopting Simar \& Wilson $(1998,2000)$ 's bootstrap procedure, the problem of testing hypothesis regarding returns to scale is conducted (Simar and Wilson, 2002). Considers the convexity constraint in (1), $\sum_{j=1}^{n} \lambda_{j}=1$, for assuming variable returns-to-scale. The above VRS DEA model gives us the result of 
TE_VRS. Next, we can easily have a constant returns-to-scale (CRS) DEA model by relaxing the convex constraint in (1). Similarly, the CRS DEA model gives us the result of TE_CRS. Then, we apply Simar and Wilson's (2002) returns-to-scale tests, bootstrapping statistics $\widehat{w}=\sum_{j=1}^{J} \theta_{j}^{C R S} / \sum_{j=1}^{J} \theta_{j}^{V R S}$, where $\theta_{j}^{C R S}$ and $\theta_{j}^{V R S}$ are technical efficiency scores under the assumptions of CRS and VRS, respectively (see also Bogetoft and Otto, 2010 and Besstremyannaya, 2013).

\subsubsection{The double bootstrap proposed by Simar \& Wilson (2007)}

Following Algorithm 2 developed by Simar and Wilson (2007), the modified double bootstrap procedure for input-oriented VRS DEA efficiency is as follows.

Step 1: Using (1) to estimate DEA input-oriented technical efficiency for all the studied white-leg shrimp farms. Next, calculate the technical efficiency score, $\widehat{\delta}_{J}=1 / \widehat{\theta}_{j}$, for the $j^{\text {th }}$ farm. The score is ranged from one to infinity.

Step 2: Estimate $\hat{\beta}$ of $\beta$ as well as $\widehat{\sigma_{\varepsilon}}$ of $\sigma$ in the truncated regression of $\widehat{\delta}_{J}$ on $Z_{j}$ when $\widehat{\delta}_{J}>1$ using maximum likelihood in which $Z_{j}$ is the vector of socio-economic and farm characteristic variables of white-leg shrimp farms defined in Table 2.

(i) Step 3: Repeat the following four steps (i-iv) $L_{l}$ to yield a set of bootstrap estimates $B_{j}=$ $\left\{\widehat{\delta_{j b}^{*}}\right\}_{b=1}^{L_{1}}$. For each $j=1, \ldots, n, \varepsilon_{j}$ is drawn from $N\left(0, \widehat{\sigma_{\epsilon}}\right)$

(ii) $\quad$ For each $j=1, \ldots, n$, compute $\delta_{j}^{*}=Z_{j} \hat{\beta}+\varepsilon_{j}$.

(iii) Construct a pseudo data set $\left(X_{j}^{*}, Y_{j}^{*}\right)$ where $X_{j}^{*}=\left(\widehat{\delta}_{J} / \delta_{j}^{*}\right) X_{j}$ and $Y_{j}^{*}=Y$.

(iv) Using the pseudo data set and (1), calculate pseudo efficiency estimates $\widehat{\delta_{J}^{*}}=1 / \widehat{\theta}_{j}^{*}$ for all $j=$ $1, \ldots, n$. 
Step 4: For each $j=1, \ldots, n$, calculate the bias-corrected estimator $\widehat{\delta}_{J}=\widehat{\delta}_{J}-\operatorname{bias}\left(\widehat{\delta}_{J}\right)$ where the bias term is $\operatorname{bias}\left(\widehat{\delta}_{J}\right)=\left(\frac{1}{L_{1}} \sum_{b=1}^{L_{1}} \widehat{\delta_{J b}^{*}}\right)-\widehat{\delta}_{J}$. The confidence intervals of the technical efficiency scores are calculated as presented in Simar \& Wilson (2000).

Step 5: Regress $\hat{\hat{\delta}}$ on $Z_{j}$ to calculate estimates $\hat{\hat{\beta}}$ and $\widehat{\sigma_{\varepsilon}}$ adopting truncated maximum likelihood.

Step 6: Repeat the following three steps (i-iii) $L_{2}$ time to generate a set of bootstrap estimates $K=$ $\left\{\left(\widehat{\widehat{\beta}^{*}}, \widehat{\sigma_{\varepsilon}^{*}}\right)_{b}\right\}_{b=1}^{L_{2}}$

(i) For each $j=1, \ldots, n, \varepsilon_{j}$ is drawn from $N\left(0, \widehat{\widehat{\sigma}_{\epsilon}}\right)$.

(ii) For each $j=1, \ldots, n$, compute $\delta_{j}^{* *}=Z_{j} \widehat{\widehat{\beta}}_{J}+\varepsilon_{j}$.

(iii) $\quad$ Regress $\delta_{j}^{* *}$ on $Z_{j}$ to yield estimates $\widehat{\widehat{\beta}^{*}}$ and $\widehat{\sigma_{\varepsilon}^{*}}$ adopting truncated maximum likelihood.

Step 7: The estimates $\hat{\hat{\beta}}$ and $\widehat{\sigma_{\varepsilon}}$ generated in Step 5 and the bootstrap estimates $K$ are used to construct confidence intervals for $\beta$ and $\sigma_{\varepsilon}$. The $(1-\alpha)$ percent confidence interval of the $j^{\text {th }}$ element of vector $\beta$ is constructed as the $\operatorname{Pr}\left(-b_{\frac{\alpha}{2}} \leq \widehat{\widehat{\beta}_{J}^{*}}-\widehat{\widehat{\beta}_{J}} \leq-a_{\frac{\alpha}{2}}\right) \approx 1-\alpha$ such that the estimated confident interval for $\beta_{j}$ is $\left[\widehat{\hat{\beta}}_{J}+a_{\alpha / 2}^{*}, \widehat{\widehat{\beta}}_{J}+b_{\alpha / 2}^{*}\right]$.

Two main points should be considered for applying the double bootstrap as follows. Firstly, considering that steps 3 and 4 in 2.2.3 (Algorithm 2) employ a parametric bootstrap in the first-stage problem in order to produce bias-corrected estimates of technical efficiency, $\widehat{\hat{\delta}}_{\mathrm{J}}$. The parametric structure assumed in this Algorithm, $\varphi\left(z_{j}, \beta\right)=z_{j} \beta$, is the simplify of the smoothing that was employed in Simar and Wilson (2000); otherwise, the bootstrap used to obtain $\widehat{\hat{\delta}_{J}}$ similar to the one described in Simar and Wilson (2000). The difference between two methods for the estimates of technical efficiency is that the single bootstrap is based only on the input and output information while the double bootstrap adjusts the estimates, based on not only this information but also the socio-economic and farm characteristic variable information. This idea has a 
link with the parametric approach for technical analysis, stochastic production function, proposed by Battese and Coelli (1995).

Secondly, for explaining the sources of farm level efficiency, Algorithm 2 adopts the reciprocal of technical

efficiency value, $\widehat{\delta}_{J}=1 / \widehat{T E}_{j}$, as dependent variable for the second-stage with truncated regression such as:

$$
\widehat{\delta_{J}}=\beta Z_{j}+\varepsilon_{j} \geq 1
$$

where $\widehat{\delta_{\mathrm{J}}}$ is, called the technical efficiency score (see also input efficiency score developed by Shephard (1970) with a distance function), with the range from 1 to infinity and $Z j$ is a vector of individual farm characteristics assumed to affect the choice and use of output and inputs, $\beta$ is a vector of parameters to be estimated, and $\varepsilon_{\mathrm{j}}$ is a continuous i.i.d. random variable, distributed as $\mathrm{N}\left(0, \sigma_{\epsilon}^{2}\right)$ with left truncation at $1-$ $\beta Z_{j}$ for each farm $j$, and assumed to be independent of $Z j$. Adopting the truncated regression for (2), this procedure avoid boundary problems since the estimated value of technical efficiencies are typically defined on the interval $[0,1]$, with, in general, few values, if any, close to 0 but some values of 1 (see Burgress and Wilson, 1998 for further discussion). Johnson and Kuosmanen (2012) emphasized that Simar and Wilson (2007) advocated the use of the truncated regression model that takes into account explicitly the bounded domain of the DEA efficiency estimates.

\subsection{Study sites and data for analysis}

The south-central coast has been considered as the major region for developing white-leg shrimp farming in Vietnam since the early 2000s. The farming activities in this region occupy the area of 8,719 ha, and the output of 43,585 tons (the productivity reaching 5 tons/ha) in the year of 2014. We select Quang Ngai, Phu Yen, Khanh Hoa and Ninh Thuan provinces for study sites. This is because white-leg shrimp is raised mainly in these areas and these account for $79.4 \%$ and $7.5 \%$ of the farming area of the south-central coast and of the whole country. Specifically, the areas for intensive farming of white-leg shrimp in Quang Ngai, Phu Yen, Khanh Hoa and Ninh Thuan are 426, 450, 698 and 900 ha, respectively (Long et al., 2016). 
The survey was was carried out in 2015 and in four regions of the south-central coast of Vietnam: Quang Ngai, Phu Yen, Khanh Hoa, Ninh Thuan. 318 intensive farming households are randomly selected for interview based on the lists of farmers obtained from the Fisheries Directorate of these provinces. Specifically, Quang Ngai has 62 households (accounting for $24 \%$ of the total number of intensive shrimp farming households in Quang Ngai province); Phu Yen has 59 households (24\%), Khanh Hoa has 95 households (22\%) and Ninh Thuan has 102 households (24\%). Each farming household, represented by the owner and/or manager, was face-to-face interviewed by two research team members from the Faculties of Economics and Aquaculture, Nha Trang University and by representatives from the Fisheries Directorate of these provinces. Data were collected using a project-designed questionnaire focusing socioeconomic characteristics, farm-specific information, outputs produced, and inputs used for the operating year of 2014. A pilot study was first conducted to validate the questionnaire and the necessary adjustments and changes were made.

There are a few small steps have been made toward introducing noise into nonparametric production models (Simar \& Zelenyuk, 2011; Simar \& Wilson, 2013; and Olesen \& Petersen, 2016). In the presence of noise, envelopment estimators could behave dramatically since they are very sensitive to extreme observations that might result only from noise (Simar, 2007). For detection of outliers, which can have large influence on the estimated DEA frontier, the super efficiency test developed by Andersen and Petersen (1993) is used. For a more thorough evaluation on detection of outliers using the super efficiency test, see Banker \& Chang (2006) and Bogetoft \& Otto (2010).

The outputs and inputs for DEA framework in this study were selected based on informed choice from the existing literature for shrimp farming (see Alam \& Murshed-e-Jahan, 2008; Kiet and Fisher, 2014; Long et al., 2016; and Hai Au et al., 2018). The production technology has, therefore, been considered to be comprising of one output and five main inputs. Five most important inputs, occupied $97.6 \%$ of production cost on average, include labor, seed, feed, chemicals/drugs and power which were assumed to adequately represent the white-leg shrimp production technology in the sample areas. Because intensive shrimp 
farming uses a variety of drugs and chemicals, the cost of chemicals and drugs (for simplicity, hear after called chemicals) is used this study. The output is harvested shrimp in kilogram (see Table 2 for details).

[Insert Table 2 about here]

Table 2 also includes variables used to investigate the determinants of technical efficiency. The socioeconomic and farm characteristics for white-leg shrimp farming practices in the south-central coat of Vietnam are: (i) socio-economic characteristics of the farm's decision maker such as education, experience, family number, technical training, occupation status; and (ii) farm characteristics including the length of shrimp farming per year, farm size and having access to formal credit. Other variables such as gender of the decision maker, climatic changes (such as salinity intrusion effect, flooding effect), water management and so on would perhaps be of importance in determining their impact on technical efficiency, but the survey information collected lacked data on these factors.

\section{EMPIRICAL RESULTS AND DISCUSSION}

The rDEA package developed by Simm and Besstremyannaya (2016) is employed in this study for the single bootstrap DEA with $B=2000$ interactions. The problem of testing hypothesis regarding returns to scale is conducted by the rDEA package with 100 interactions. This package is also used for double bootstrap DEA model with $L_{1}=100$ interactions for the first loop and $L_{2}=2000$ for the second loop of Algorithm 2. The similar result can be also obtained by the simarwilson routine for Algorithm 2 developed by Badunenko \& Tauchmann (2019) from STATA software. For comparison, the conventional two-stage DEA analysis with Tobit regression is also conducted for this aquaculture. We used the Benchmarking package created by Bogetoft and Otto (2010) for the conventional two-stage DEA approach. Both rDEA and Benchmarking packages are linked to the $R$ Project for Statistical Computing.

\subsection{Descriptive statistics of the data}


Table 3 presents the summary statistics of the data collected in the study. Regarding socio-economic variables, the average experience of the sample decision makers is 9.8 years; 17 percent of the farm decision makers have the educational level of college or higher; 51 percent have participated in extension services; 24 percent of farms have access to formal credit. There are $53 \%$ of the sample decision makers working part-time business in shrimp farming and $47 \%$ of them working full-time. Average of the household number is 3.5 persons (min. 2 persons; max. 7 persons). Average farm size is 0.76 ha ranging from 0.18 to 6 ha. The mean of culture length in the year is 185 days with min of 55 and max of 330 days.

[Insert Table 3 about here]

The mean shrimp output harvested is $23211 \mathrm{~kg}$ per ha per year (min. $2000 \mathrm{~kg}$; max. $50010 \mathrm{~kg}$ ). Labor input is measured as the number of man-hours per ha for various activities, and it includes all hired and family labor, assuming that 1 day consists of 8 working hours. The mean number of hours spent per ha per year is 6429 (min. 1200 hours; max. 16000 hours). Seed is measured as the physical quantity of seeds in 1000 individuals per ha per year. There are on average 3337 thousand post-larvae (i.e., thousand individual shrimp) per ha (min. 500; max. 9900). Feed is one of the most important components of aquaculture and constitutes more than $50 \%$ of production cost. The amount of feed used is measured in $\mathrm{kg}$. The average quantity of feed used by the sample farmers is $33517 \mathrm{~kg}$ per ha per year. The mean of chemicals and drugs cost used for a year is 282512 thousand VND per ha. Finally, power devoted to shrimp production is measured in Kwh of electrics per ha. The average number of electrics spent per year is $224140 \mathrm{Kwh}$.

\subsection{Technical efficiency estimation}

Table 4 presents Simar and Wilson's (2002) test for returns-to-scale of production technology in white-leg intensive shrimp farming in the south-central coast of Vietnam. The test demonstrates that the null hypothesis of CRS is rejected in favour of VRS. Consequently, in our analysis, we estimate DEA models for VRS returns-to-scale. Next, Table 5 illustrates estimated levels of efficiency for conventional, single and double bootstrapping approaches. Over all farms, conventional DEA estimate model for average 
technical efficiency is 0.77 . This means that, on average, white-leg shrimp farming households can reduce their inputs by $23 \%$ without changing the level of their output.

[Insert Table 4 about here]

From these initial estimates, we apply both methods of bootstrapping to correct for the bias of technical efficiency: the single bootstrap in Simar and Wilson (2000) and the double bootstrap in Simar and Wilson (2007). The two bootstrapping methods reveal similar results. The average bias-corrected estimate for the single bootstrap is 0.67 while the one for the double bootstrap is 0.69 . The correlation between the two biascorrected estimates (i.e., single and double bootstrap) is 0.91; the Spearman rank correlation is 0.91. This means that the efficiency ranking of the shrimp farms are consistent in both bootstrap methods. In addition, the width of the $95 \%$ confidence intervals is 0.17 for technical efficiency using the single bootstrap method and 0.10 for the double bootstrap method. This indicates a lower statistical variability of DEA efficiency estimate using the double bootstrap. This result may be because the single bootstrap method is based only on the input and output information while the double bootstrap adjusts the estimates, based on not only this information but also the socio-economic and farm characteristic variable information. This is also the case in Balcome et al. (2008).

\section{[Insert Table 5 about here]}

The lower and upper bounds of the $95 \%$ confidence interval for the bias-corrected technical efficiency in the double bootstrap are 0.65 and 0.75 , respectively, which suggests that the amount an "average" farm could save its input by increased technical efficiency ranged from $25 \%$ to $35 \%$. Clearly, as a result of estimating the bias-corrected measures and interval estimates of technical efficiency, our results can be viewed by policy makers with increased confidence.

[Insert Table 6 about here] 
As the efficiencies estimated from the conventional and double bootstrap DEA are not independent, we use the paired difference $t$-test, the two-sample Kolmogorov-Smirnov test, and the Kruskal-Wallis rank sum test to compare the estimates (see Bogetoft \& Otto, 2010 for details). The results are presented in Table 6. Based on these tests, on average, the technical efficiency estimated from the conventional DEA is statistically significantly higher than in the double bootstrap method. Latruffe et al. (2008) and Olson \& Vu (2009) also found the same relationship in their agricultural studies. The results in Table 5 show that the biases in the uncorrected results are rather substantial. Specifically, the conventional estimate of 0.77 means that with a given output, an average farm could reduce its input by $23 \%$ if the technical efficiency value were improved to 1 . The bias-corrected estimate of 0.69 , however, suggests an expected input reduction of $31 \%$ for the double bootstrap estimate. It is clear that the percentage of input saving is considerably greater using the double bootstrapping compared to the conventional approaches for the case of white-leg shrimp aquaculture in the central south coast of Vietnam.

\subsection{Determinants of technical efficiency}

From a policy perspective, the estimates of efficiency propose that there is considerable room for improvement in technical efficiency in the intensive white-leg shrimp farming in Vietnam. Thus, it would appear sensible to examine the determinants of technical efficiency for this case.

\section{[Insert Table 7 about here]}

For robust checking, the Tobit regression in the second stage of the conventional DEA approach is conducted. To be consistent with the double bootstrap method, the dependent variable in the Tobit regression is also the reciprocal of the estimates of technical efficiency in (1). Therefore, a positive relationship between a shrimp farm specific variable and technical efficiency exists if the sign of the coefficient is negative, and negative if the coefficient is positive (see also Balcombe et al., 2008).

[Insert Table 8 about here] 
Our estimation reveals some interesting findings. First of all, educated shrimp farmers can be expected to comprehend information and have better management skills, thereby making them more technically efficient. The variable of education is positively related to a farm's technical efficiency in the double bootstrap estimation, although the relationship is very weak. This is also the cases of aquaculture farms in practices such as in India by Roy and Rens (2008); in Bangladesh by Iliyasu et al. (2016); and in Vietnam by Lam Anh et al. (2018). In contrast, the Tobit regression for a conventional estimation displays the unexpected sign for this variable. It is, however, not statistically significant at the $90 \%$ confidence interval.

Second, it is expected that the shrimp farmers with greater experience are more technically efficient than those who have little experience. This can be explained by the learning-by-doing hypothesis (see Nilsen, 2010 for an example). The coefficients estimated for the experience have a positive impact on technical efficiency for both estimations. However, the estimate is rather statistically weak for both models. This is also found in some aquaculture practices such as in Bangladesh by Alam (2011); and in China by Yin et al. (2014) and Zongli et al. (2014).

The third explanatory variable is technical training from extension agents. Contrary to our expectation, the technical training is recognized to have a negative impact on technical efficiency for both estimations, although the relationship is very weak. This may implies that shrimp farmers who receive technical training from extension agents may be less technically efficient than those who rely only on their lengthy experience. Our result is in line with Redy et al. (2008) for shrimp aquaculture in India and Kiet \& Fisher (2014) for shrimp farming in the Mekong river delta of Vietnam. Technical training from extension is, even, found a statistically significant negative effect on technical efficiency, which is explained by the inappropriate training for the case of shrimp farming in the Mekong river delta of Vietnam (Kiet \& Fisher, 2014). Our result may be explained by a large number of households in our sample (51\%) who have attended extension training courses. However, the quality of the extension training for shrimp aquaculture the south-central coast of Vietnam may be re-assessed. 
The estimated coefficients of the occupation status showed that farmers who operate intensive shrimp farming as a full-time business may be more efficient than part-time farmers in both models even though statistically insignificant at the level of $10 \%$. Observation from the survey reveals that most of the farmers who operate shrimp farming as a part-time business have alternative sources of income and tend to put less energy and resources in their shrimp farms; hence they may be less efficient. This is also found in the case of aquaculture in Malaysia by Iliyasu et al. (2016).

The farm size has a significant positive impact on technical efficiency in both double bootstrap and Tobit estimations within the $95 \%$ confidence interval. The positive relationship between farm size and technical efficiency indicates that the larger the farm size is, the more efficiently the farmers utilize their inputs. More often than not, large farms tended to reap the benefits of economies of scale (see also Iliyasu et al., 2014). This is in line with the findings reported by some aquaculture studies such as Dey et al. (2005) for intensive freshwater pond poly-culture production in Vietnam; Yin et al. (2014) for carp farming in China; Thap et al. (2016) for white-leg shrimp farming in Ninh Thuan, Vietnam; and Hai Au et al. (2018) for lobster aquaculture in Vietnam.

We also find a positive relationship between having access to formal credit for operational cost and technical efficiency. This estimate is statistically significant at the level of 5\% for the double bootstrap. The relationship is also found in the tobit regression with the confident level of $90 \%$. The result implies that the shrimp farms which are able to access to formal credit are more technically efficient than others. The positive relationship between credit availability and cost efficiency is also found in Cinemre et al. (2006) for the case of trout farming in the Black Sea Region, Turkey. Mitra et al. (2019) showed that aquaculture productivity is significantly higher for farmers who are not exposed to credit constraints in Bangladesh. Rahman et al. (2019) also showed the negative association of technical efficiency with credit constraints. The field survey reveals that the intensive white-leg shrimp farming in the south-central coast of Vietnam requires high operational expenses (see Long et al. 2016). Shrimp farm households with a loan for operational costs from input suppliers and even unofficial financial markets often bear high costs for 
receiving credit with tight schedules for paying the loans off. The shrimp farmers with credit constraints are, therefore, not able to provide the right amount of fingerlings to the ponds, purchase formulated feed or sell the shrimp at the optimal size, which influences efficiency negatively. There is, however, only $24 \%$ of households having access to formal credit for operational cost in our sample (see Table 3). Moreover, it is not easy for shrimp farm households to get the official credits from commercial banks because of (i) limited assets for mortgage and (ii) complicated borrowing procedures for a high risky farming.

As expected, cultivation length in a year has a significant negative impact on technical efficiency in both models within the $95 \%$ confidence interval. This may be due to two reasons. Firstly, farmers who keep their shrimp longer than necessary are more likely to use greater inputs with little or no gain in additional output, thus becoming inefficient (see also Iliyasu et al., 2014; Thap et al., 2016; and Zongli et al., 2017). Secondly, farmers with longer culture period (intensive use of land) may face higher risks of the spread of shrimp disease and pollution. They are, therefore, likely becoming inefficient.

The provincial effect may be important for technical efficiency of intensive shrimp farming in the study area. If other variables keep unchanged, the result in both models implies that a shrimp farm in Phu Yen is statistically more technical efficient than a farm in Quang Ngai in the double bootstrap model with the significant level of 5\%. The relationship is also found in the tobit regression with the confident level of $90 \%$. This result might be explained by other factors which are not captured in the regression models such as weather, land quality, or water quality.

Overall, the comparison of the double bootstrap estimation in Table 7 with Tobit estimation using non bias corrected scores in Table 8 shows only slight differences. However, stronger relationships result from the application of Algorithm 2 in Simar \& Wilson's (2007). This trend is hold in Latruffe et al. (2008) for the case of agriculture farming in the Czech Republic. Balcombe et al. (2008) also concludes that their findings employed the double bootstrap DEA do not contradict previous studies adopted the conventional two-stage 
DEA for the case of rice farming in Bangladesh. Olson \& Vu (2008) showed that while results from both methods are not substantively for the case of agriculture farms in US.

\section{CONCLUSIONS}

The paper adopts advantages of bootstrapping proposed by Simar \& Wilson (1998, 2000 and 2007) into DEA framework to make statistical inferences about the technical efficiency, which can be viewed by policy makers with increased confidence to draw broad policy implications. The intensive white-leg shrimp farming in the south-central coast of Vietnam is used as an illustrative case. Firstly, we apply Simar and Wilson's (2002) returns-to-scale test, based on the bootstrap procedure proposed by Simar \& Wilson (1998), to raise the precision in the measurement of the boundary of the production possibilities set in the non-parametric analysis. The result demonstrates that the null hypothesis of CRS is rejected in favour of VRS for the production technology. Secondly, the single bootstrap proposed Simar \& Wilson $(1998,2000)$ and the double bootstrap in Simar \& Wilson (2007) are employed to determine the variability in technical efficiency estimates and to correct for the bias inherent in the conventional measurement. The result shows that the potential improvement in the technical efficiencies suggested by both bootstrapping methods in this study is therefore certainly greater than that using conventional DEA, which has been adopted widely in the aquaculture literature. Moreover, there is a lower statistical variability of DEA efficiency estimate using the double bootstrap. Specifically, the double bootstrap DEA shows that the bias-corrected point estimate of technical efficiency is 0.69 , and at the $95 \%$ confidence interval is estimated to be 0.65 at the lower limit and 0.75 at the upper limit. This suggests that there is considerable room for improvement in technical efficiency in the intensive white-leg shrimp farming in Vietnam.

The analysis also reveals that an increased farm size and having access to formal credit in intensive shrimp farming could enhance technical efficiency. The variable that is negatively related to technical efficiency, and hence hampers farm performance, is a longer cultivation period. The result suggests that policies for better access to formal credit and land are the leading constraints on improved productivity in this shrimp 
farming. To help farmers avoid a long shrimp cultivation than necessary and identify an optimal harvesting strategy, market information on shrimp output should be provided by the government. Further development of intensive shrimp farming in the south-central coast of Vietnam should be undertaken with care of risks of the spread of shrimp disease and pollution. From a methodological point of view, the slightly different results derived from both the double bootstrap estimation and the Tobit regressed indicate that the identified factors are convincing. Nevertheless, stronger relationships result from the application of Simar and Wilson's (2007) algorithm. Therefore, we can, as a result of the methods employed in this article, feel very secure in presenting these findings. In addition, this result also implies that the findings of previous studies, employing a conventional two-step DEA in aquaculture, may largely remain valid.

It can be also stressed that the developments in DEA methodology examined in this article have been applied by few studies in aquaculture to date. There is clearly a need for greater adoption and consideration of the methods employed here to provide increased insight into their potential for the aquaculture studies where statistical inference for broad policies are very important. Although there is the slightly different result derived from both the double bootstrap estimation and the Tobit regressed in this study, it is advisable to use Algorithm 2 of the Simar and Wilson (2007) double bootstrap procedure with a well-defined, coherent statistical model in further research on technical efficiency in aquaculture, as it can increase the confidence that policy makers can place on results generated

Last but not least, in frontier analysis, the nonparametric approach DEA is based on envelopment ideas which assume that with probability one, all observed units belong to the attainable set. In these deterministic frontier models, statistical inference is now possible, by using bootstrap procedures. However, no noise is considered in the data generating process in the bootstrap procedures proposed by Simar \& Wilson in 1998, 2000 \& 2007 (see Simar, 2007; Simar \& Zelenyuk, 2011). In the presence of noise, envelopment estimators could behave dramatically since they are very sensitive to extreme observations that might result only from noise. Due to stochastic nature of the aquaculture, future studies should consider some procedures, e.g 
proposed by Simar (2007), Simar \& Zelenyuk (2011) and so on, which introducing the noise in nonparametric frontier models into industry such as (see Olesen \& Petersen, 2016).

FUNDING We would like to thank the B2014-13-12 Project from Vietnam's Ministry of Education and Training and the European Union's Horizon 2020 Project ClimeFish 677039 for financial support.

\section{REFERENCES}

Alam, F. (2011). Measuring technical, allocative and cost efficiency of pangas (Pangasius hypophthalmus: Sauvage 1878) fish farmers of Bangladesh. Aquaculture Research, 42(10), 1487-1500.

Alam, F., \& Murshed-e-Jahan, M., 2008. Resource allocation efficiency of the prawn-carp farmers of Bangladesh. Aquaculture Economics \& Management, 12(3), 188-206.

Amos, T. T. (2007). Production and productivity of Crustacean in Nigeria. Journal of Social Science, 15(3), 229-233.

Anh Ngoc, P. T., Gaitán-Cremaschi, D., Meuwissen, M. P., Le, T. C., Bosma, R. H., Verreth, J., \& Lansink, A. O. (2018). Technical inefficiency of Vietnamese pangasius farming: A data envelopment analysis. Aquaculture economics \& management, 22(2), 229-243.

Anh, P. T., Kroeze, C., Bush, S. R., \& Mol, A. P. (2010). Water pollution by intensive brackish shrimp farming in south-east Vietnam: Causes and options for control. Agricultural Water Management, 97(6), 872-882.

Asche, F., Guttormsen, A. G., \& Nielsen, R. (2013). Future challenges for the maturing Norwegian salmon aquaculture industry: An analysis of total factor productivity change from 1996 to 2008. Aquaculture, 396, 43-50.

Badunenko, O., \& Tauchmann, H. (2019). Simar and Wilson two-stage efficiency analysis for Stata. The Stata Journal (Accepted/In press).

Badunenko, O., \& Mozharovskyi, P. (2016). Nonparametric frontier analysis using Stata. The Stata Journal, $16(3), 550-589$. 
Balcombe, K., Fraser, I., Latruffe, L., Rahman, M., \& Smith, L. (2008). An application of the DEA double bootstrap to examine sources of efficiency in Bangladesh rice farming. Applied Economics, 40(15), $1919-1925$.

Battese, G.E. and T. Coelli (1995), A model for technical inefficiency effects in a stochastic frontier production function for panel data. Empirical Economics, 20, 325-332.

Besstremyannaya, G. (2013). The impact of Japanese hospital financing reform on hospital efficiency: A difference-in-difference approach. The Japanese Economic Review, 64(3), 337-362.

Bogetoft, P., \& Otto, L. (2010). Benchmarking with DEA, SFA, and R (Vol. 157). Springer Science \& Business Media, New York, USA.

Chang, H., Boisvert, R.N., \& Hung, L. (2010). Land subsidence, production efficiency and the decision of aquaculture firms in Taiwan to discontinue production. Ecological Economic, 69(12), 2448-2456.

Cinemre, H. A., Ceyhan, V., Bozoglu, M., Demiryurek, K., \& Kilic, O. (2006). The cost efficiency of trout farms in the black sea region, Turkey. Aquaculture, 251(2), 324-332.

Coelli, T. J., Rao, D. S. P., \& Battese, G. E. (2005). An introduction to efficiency and productivity analysis. Plymouth, Massachusetts: Kluwer Academic Publishers.

Dey, M.M., Paraguas, F.J., Srichantuk, N., Xinhua, Y., Bhatta, R. \& Dung, C. L. T. (2005). Technical efficiency of freshwater pond polyculture production in selected Asian Countries. Estimation and implication. Aquaculture Economics \& Management, 9(1), 39-63.

Den, T. D., Ancev, T., \& Harris, M. (2007). Technical efficiency of prawn farms in the Mekong Delta, Vietnam. 51st AARES Annual Conference, February 13-16, 2007. Queenstown, NZ: Australian Agricultural and Resource Economics Society.

FAO. (2018). The State of World Fisheries and Aquaculture 2018 - Meeting the sustainable development goals. Rome, Italy.

Fernandes, F. D. S., Stasinakis, C., \& Bardarova, V. (2018). Two-stage DEA-Truncated Regression: Application in banking efficiency and financial development. Expert Systems with Applications, 96, 284-301. 
Fisheries Directorate of Vietnam. (2017). Overall scheme of developing Vietnam shrimp industry till 2030 (in Vietnamese). Ministry of Agriculture and Rural Development, Hanoi, Vietnam.

Iliyasu, A., Mohamed, Z. A., Ismail, M. M., Abdullah, A. M., Kamarudin, S. M., \& Mazuki, H. (2014). A review of production frontier research in aquaculture (2001-2011). Aquaculture Economics \& Management, 18(3), 221-247.

Iliyasu, A., \& Mohamed, Z. A. (2015). Technical efficiency of tank culture systems in peninsular Malaysia: An application of data envelopment analysis. Aquaculture economics \& management, 19(4), 372386.

Iliyasu, A., \& Mohamed, Z. A. (2016). Evaluating contextual factors affecting the technical efficiency of freshwater pond culture systems in Peninsular Malaysia: A two-stage DEA approach. Aquaculture Reports, 3, 12-17.

Iliyasu, A., Mohamed, Z. A., \& Terano, R. (2016). Comparative analysis of technical efficiency for different production culture systems and species of freshwater aquaculture in Peninsular Malaysia. Aquaculture Reports, 3, 51-57

Johnson, A.L., Kuosmanen, T. (2012). One-stage and two-stage DEA estimation of the effects of contextual variables. European Journal of Operation Research 220, 559-570.

Keramidou, I., \& Mimis, A. (2011). An application of the double-bootstrap data envelopment analysis to investigate sources of efficiency in the Greek poultry sector. World's Poultry Science Journal, 67(04), 675-686.

Kiet, N. T., \& Fisher, T. C. (2014). Efficiency analysis and the effect of pollution in the Mekong river delta. Aquaculture Economics \& Management, 18(4), 325-343.

Kim Anh, N. T., Jolly, C. M., Chương, B. N. N, \& Trang. L. T. H. (2016). Aquaculture and poverty alleviation in Ben Tre Province, Vietnam. Aquaculture economics \& management, 20(1), 82-108.

Kobayashi, M., Msangi, S., Batka, M., Vannuccini, S., Dey, M. M., \& Anderson, J. L. (2015). Fish to 2030: the role and opportunity for aquaculture. Aquaculture economics \& management, 19(3), 282-300. 
Ha, T. T. P., van Dijk, H., Bosma, R., \& Sinh, L. X. (2013). Livelihood capabilities and pathways of shrimp farmers in the Mekong Delta, Vietnam. Aquaculture economics \& management, 17(1), 1-30.

Ha, T. T. T., \& Bush, S. R. (2010). Transformations of Vietnamese shrimp aquaculture policy: empirical evidence from the Mekong Delta. Environment and Planning C: Government and Policy, 28(6), 1101-1119.

Hai Au, T. N., The, B. D., \& Speelman, S. (2018). Analyzing the variations in cost-efficiency of marine cage lobster aquaculture in Vietnam: A two-stage bootstrap DEA approach. Aquaculture Economics \& Management, 22(4), 458-473.

Hassanpour, B., Ismail, M. M., Mohamed, Z., \& Kamarulzaman, N. H. (2010). Sources of productivity growth in rainbow trout aquaculture in Iran: technical efficiency change or technological progress?. Aquaculture Economics \& Management, 14(3), 218-234.

Latruffe, L., Davidova, S., \& Balcombe, K. (2008). Application of a double bootstrap to investigation of determinants of technical efficiency of farms in Central Europe. Journal of Productivity Analysis, 29(2), 183-191.

Long, L. K., \& Binh, P. T. (2013). Profitability analysis for white leg shrimp aquaculture farms in Ninh Hoa town, Khanh Hoa province (in Vietnamese). Journal of Fisheries Science and Technology, 2, 62-66, Nha Trang University, Vietnam.

Long, L. K., Thap, L. V., Thuy, P. T. T., \& Thuy, N. X. (2016), Sustainable development of white-leg shrimp farming in the Vietnam's southern central coastal provinces (in Vietnamese), Vietnam's Ministry of Education and Training Project (code: B2014-13-12), University of Nha Trang, Khanh Hoa, Vietnam.

Lam Anh, N. P., T. B., Bosma, R., Verreth, J., Leemans, R., De Silva, S., \& Lansink, A. O. (2018). Impact of Climate Change on the Technical Efficiency of Striped Catfish, Pangasianodon hypophthalmus, Farming in the Mekong Delta, Vietnam. Journal of the World Aquaculture Society, 49(3), 570-581.

Mitra, S., Khan, M. A., \& Nielsen, R. (2019). Credit constraints and aquaculture productivity. Aquaculture Economics \& Management, 1-18. 
Nhuong, Tr., Bailey, C., Wilson, N., \& Phillips, M. (2013). Governance of global value chains in response to food safety and certification standards: The case of shrimp from Vietnam. World Development, 45, 325-336.

Nilsen, O. B. (2010). Learning-by-doing or technological leapfrogging: A production frontier and efficiency measurement in Norwegian salmon aquaculture. Aquaculture Economics \& Management, 14(2), 97-119.

Nielsen, R. (2011). Green and technical efficient growth in Danish fresh water aquaculture. Aquaculture Economics \& Management, 15(4), 262-277.

Nielsen, R. (2012). Introducing individual transferable quotas on nitrogen in Danish fresh water aquaculture: Production and profitability gains. Ecological Economics, 75, 83-90.

Nielsen, R., Andersen, J. L., \& Bogetoft, P. (2014). Dynamic reallocation of marketable nitrogen emission permits in Danish freshwater aquaculture. Marine Resource Economics, 29(3), 219-239.

Olesen, O. B., \& Petersen, N. C. (2016). Stochastic data envelopment analysis-A review. European Journal of Operational Research, 251(1), 2-21.

Olson, K., \& Vu, L. (2009). Economic efficiency in farm households: Trends, explanatory factors, and estimation methods. Agricultural Economics, 40(5), 587-599.

Onumah, E. E. (2010). Elements which delimitate technical efficiency of fish farms in Ghana. Journal of the World Aquaculture Society, 41(4), 506-513.

Rahman, M. T., Nielsen, R., Khan, M. A., \& Asmild, M. (2019). Efficiency and production environmental heterogeneity in aquaculture: A meta-frontier DEA approach. Aquaculture, 509, 140-148.

Reddy, G.P., M.N. Reddy, B.S. Sontakki, \& D.B. Prakash (2008) Measurement of efficiency of shrimp (peneaus monodon) farmers in Andhra Pradesh. Indian Journal of Agricultural Economics, 63(4), $653-657$.

Roy, A. K., \& Jens, N. (2008). Econometric approach for estimation of technical efficiency of aquaculture farms. In Roy, A. K., \& Serangi, N. (Eds.), Applied bioinformatics, statistics and economics in fisheries research (pp. 501-518). New Delhi: New India Publishing Agency. 
Sharma, K. R., \& Leung, P. (2003). A review of production frontier analysis for aquaculture management. Aquaculture Economics \& Management, 7(1-2), 15-34.

Shephard, R.W. (1970). Theory of Cost and Production Function. Princeton University Press, Princeton.

Simar, L. (2007). How to improve the performances of DEA/FDH estimators in the presence of noise?. Journal of Productivity Analysis, 28(3), 183-201.

Simar, L. \& Wilson, P. (1998). Sensitivity Analysis of Efficiency Scores: How to Bootstrap in Nonparametric Frontier, Management Science, 44(1), 49-61.

Simar, L., \& Wilson, P. (2000). Statistical inference in nonparametric frontier models. The state of the art. Journal of Productivity Analysis, 13(12), 49-78.

Simar, L., \& Wilson, P. W. (2002). Non-parametric tests of returns to scale. European Journal of Operational Research, 139(1), 115-132.

Simar, L., \& Wilson, P. W. (2007). Estimation and inference in two-stage, semi-parametric models of production processes. Journal of Econometrics, 136(1), 31-64.

Simar, L., \& Wilson, P. W. (2011). Two-stage DEA: caveat emptor. Journal of Productivity Analysis, 36(2), 205.

Simar, L., \& Wilson, P. W. (2013). Estimation and inference in nonparametric frontier models: Recent developments and perspectives. Foundations and Trends® in Econometrics, 5(3-4), 183-337.

Simar, L., \& Zelenyuk, V. (2011). Stochastic FDH/DEA estimators for frontier analysis. Journal of Productivity Analysis, 36(1), 1-20.

Simm, J., \& Besstremyannaya, G. (2016). Package 'rDEA', (https://cran.rproject.org/web/packages/rDEA/rDEA.pdf) (accessed 31.10.18).

Sinh, X.L. (2006). Major considerations on the fishery sector in the Mekong Delta of Vietnam. Presentation to the CARD workshop, July 15-20, 2006, Cantho, Vietnam.

Singbo, A. G., \& Lansink, A. O. (2010). Lowland farming system inefficiency in Benin (West Africa): directional distance function and truncated bootstrap approach. Food Security, 2(4), 367-382. 
Singbo, A. G., Lansink, A. O., \& Emvalomatis, G. (2014). Estimating farmers' productive and marketing inefficiency: an application to vegetable producers in Benin. Journal of Productivity Analysis, 42(2), 157-169.

Tan, R.L., Y.T. Garcia, M.L. Dator, I.M.A. Tan, \& D.E. Pemsl. (2011). Technical efficiency of Genetically Improved Farmed Tilapia (GIFT) cage culture operations in the lakes of Laguna and Batangas, Philippines. Journal of the International Society of Southeast Asian Agricultural Sciences, 17(1), 194-207.

Thap, L. V., Long, L. K., \& Hoai, N. T. (2016). Analysis of technical efficiency of intensive white-leg shrimp farming in Ninh Thuan, Vietnam: An application of the double-bootstrap data envelopment analysis. IIFET 2016 Scotland Conference Proceedings, Oregon University, USA.

Theodoridis, A., Batzios, C., Ragkos, A., \& Angelidis, P. (2017). Technical efficiency measurement of mussel aquaculture in Greece. Aquaculture international, 25(3), 1025-1037.

Thuyet, B. D., Luong-Van, J., \& Austin, C. M. (2012). Impact of shrimp farm effluent on water quality in coastal areas of the world heritage-listed Ha Long Bay. American Journal of Environmental Sciences, 8(2), 104-116.

VASEP. (2013). Report for Vietnamese shrimp exports in 2012 and forecast for the year 2013 (In Vietnamese). Hanoi, Vietnam.

Vassdal, T., \& Sørensen Holst, H. M. (2011). Technical progress and regress in Norwegian salmon farming: a Malmquist index approach. Marine Resource Economics, 26(4), 329-341.

Yin, X., Wang, A., Zhou, H., Wang, Q., Li, Z., \& Shao, P. (2014). Economic efficiency of crucian carp (Carassius auratus Gibelio) polyculture farmers in the coastal area of Yancheng City, China. Turkish Journal of Fisheries and Aquatic Sciences, 14(2), 429-437.

Zongli, Z., Yanan, Z., Feifan, L., Hui, Y., Yongming, Y., \& Xinhua, Y. (2017). Economic efficiency of small-scale tilapia farms in Guangxi, China. Aquaculture Economics \& Management, 21(2), 283294. 